\title{
Power-Law Intensity Distribution of $\gamma$-Decay Cascades: Nuclear Structure as a Scale-Free Random Network
}

\author{
Keisuke Fujii $\oplus^{1,2, *}$ and Julian C. Berengut $\oplus^{3,2}$ \\ ${ }^{1}$ Department of Mechanical Engineering and Science, Graduate School of Engineering, Kyoto University, Kyoto 615-8540, Japan \\ ${ }^{2}$ Max-Planck-Institut für Kernphysik, Saupfercheckweg 1, 69117 Heidelberg, Germany \\ ${ }^{3}$ School of Physics, University of New South Wales, New South Wales 2052, Australia
}

(Received 26 May 2020; revised 17 November 2020; accepted 9 February 2021; published 12 March 2021)

\begin{abstract}
By modeling the transition paths of the nuclear $\gamma$-decay cascade using a scale-free random network, we uncover a universal power-law distribution of $\gamma$-ray intensity $\rho_{I}(I) \propto I^{-2}$, with $I$ the $\gamma$-ray intensity of each transition. This property is consistently observed for all datasets with a sufficient number of $\gamma$-ray intensity entries in the National Nuclear Data Center database, regardless of the reaction type or nuclei involved. In addition, we perform numerical simulations that support the model's predictions of level population density.
\end{abstract}

DOI: 10.1103/PhysRevLett.126.102502

The line intensity distribution for many-electron atoms (one of the most well-studied fermionic many-body systems) in plasmas has been reported to exhibit a power-law dependence [1-4],

$$
\rho_{I}(I) \propto I^{-b}
$$

where $\rho_{I}(I)$ is the number of emission lines with intensity $I$ and $b$ is the index of the power law. In our earlier work [5], we showed that the index can be written as $b=2 T_{\mathrm{ex}} / T+1$, where $T_{\mathrm{ex}}$ is the excitation temperature in the plasma (in the energy scale), and $T$ is an atom-dependent constant related to the level density of the atom [6]. This relation was derived based on two general principles: the stochastic property of the transition rates, and the exponential energy dependence of the level density of fermionic many-body systems. Therefore, we may expect other systems with the same properties, such as heavy nuclei, to exhibit a similar intensity power law. However, the relationship to other power laws observed in many diverse fields of science has not yet been elucidated [7-11].

Recently, it was shown that a random walk on a general network structure typically exhibits a power-law distribution [12-14]. For example, Corominas-Murtra et al. showed that the probability of a node being visited during a random walk on an acyclic network follows a power law $[13,14]$. In this Letter, we discuss the connection between

Published by the American Physical Society under the terms of the Creative Commons Attribution 4.0 International license. Further distribution of this work must maintain attribution to the author(s) and the published article's title, journal citation, and DOI. Open access publication funded by the Max Planck Society. quantum many-body systems and a scale-free random network such as this. Our approach is philosophically similar to Wigner's modeling of the Hamiltonian of a quantum many-body system using a random matrix (see, e.g., [15]). Here instead, we model the optical transition paths using a scale-free random network, where the levels of the quantum system correspond to the nodes of the network while the transitions correspond to the edges.

In particular, we discuss $\gamma$-decay cascades of heavy nuclei. After a nuclear reaction, such as thermal neutron capture, an excited nucleus at a particular excited level, of which the atomic number has been incremented, is generated. The excited nucleus decays to a lower level by emitting a $\gamma$-ray photon until it reaches the ground state [see Fig. 1(a) later]. A nucleus cannot be excited to any upper levels again, and thus, as the cascade proceeds, the number of levels that can decay is reduced. This is very similar to the sample-state-reducing (SSR) process discussed in Refs. $[13,14]$. We show that such a process on a scale-free random acyclic network exhibits a universal power law $\rho_{I}(I) \propto I^{-2}$, where $I$ is the probability of passing a particular edge during the cascade and $\rho_{I}$ is the number density of edges with the probability $I$. In the $\gamma$-decay cascade, $I$ corresponds to the intensities of the $\gamma$-decay transitions. We demonstrate below that all large datasets comprising $\gamma$-ray intensities that are stored in the Evaluated Nuclear Structure Data File (ENSDF) database [16] comply with this intensity power law regardless of the nuclear structure and reaction type.

We begin by considering a cascade process on an acyclic network - a directed network with no cycles - consisting of $N$ nodes [17]. Acyclic networks have a natural ordering, and the indices $1, \ldots, N$ are assigned to each node according to this ordering. The cascade process starts from the $N$ th node. Let $r_{n, m}$ be the weight of an edge from the $m$ th node to the $n$th node, and assume that the probability of 

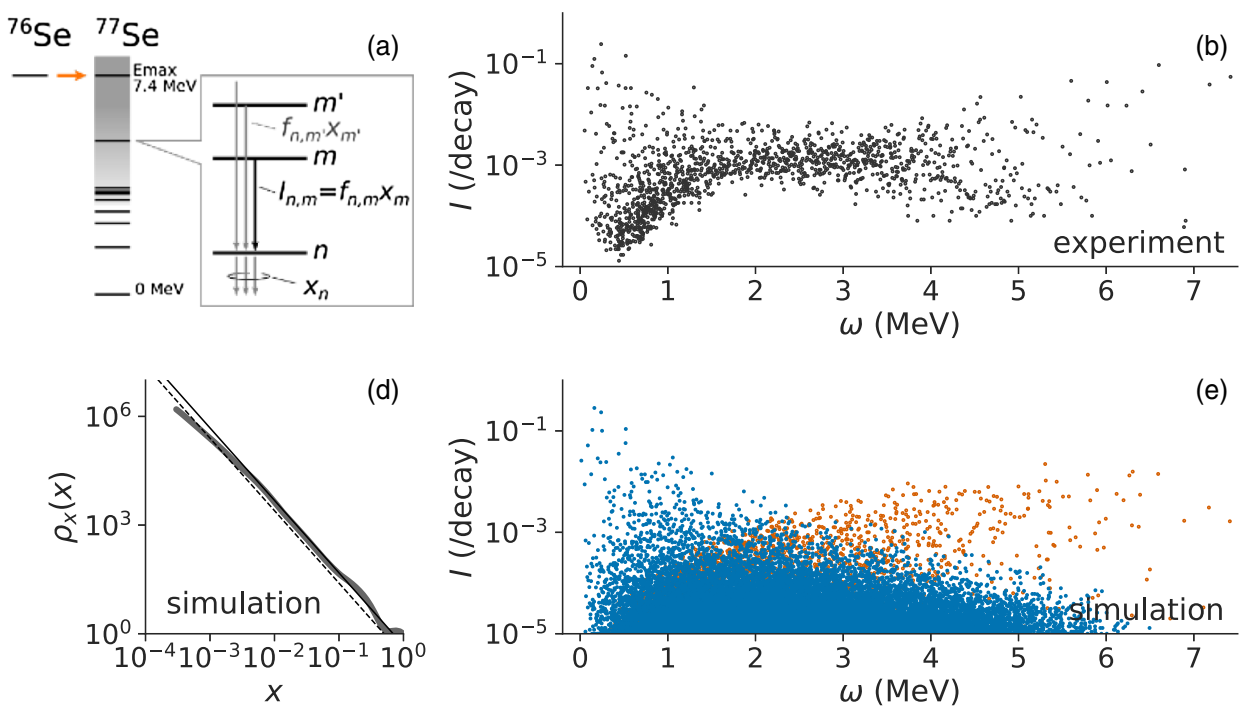
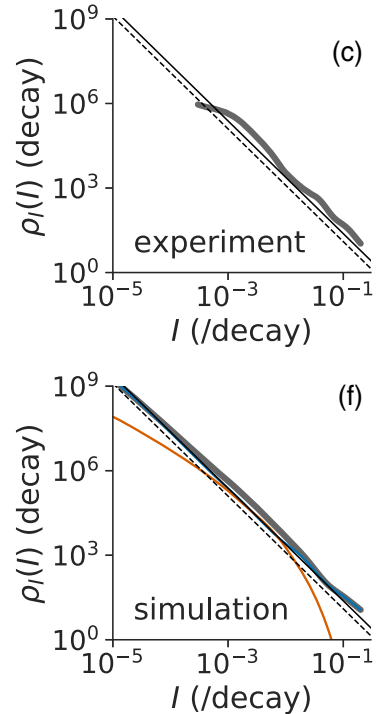

FIG. 1. (a) Schematic illustration of the $\gamma$-decay cascade after the thermal neutron capture of ${ }^{76} \mathrm{Se}$. (b),(c) Experimentally observed distributions of $\gamma$ rays resulting from this process. (d)-(f) Distributions simulated by RAINIER. (b),(e) Joint distributions of the $\gamma$-ray intensity and energy, respectively. (c),(f) Density distribution of $\gamma$-ray lines with a given intensity $I$. (d) Simulated distribution of $x$, which is the probability of experiencing a particular level during the cascade. Solid and dotted straight lines in (c), (d), and (f) show the theoretical results with $\eta=12 / 25$ and $\eta=1 / 4$, respectively. In (e) and (f), the contribution from the resonance state is shown in a different color.

jumping from the $m$ th node to the $n$th node in one step (the branching ratio) is determined from this weight as $f_{n, m}=r_{n, m} / r_{m}$, where $r_{m}=\sum_{n<m} r_{n, m}$. Note that the discussions below can be generalized to unweighted networks by assuming binary weights $r_{n, m} \in\{0,1\}$. The total probability of passing the edge $n \leftarrow m$ during the cascade can be written as

$$
I_{n, m}=f_{n, m} x_{m},
$$

where $x_{m}$ is the total probability of the decay passing through the $m$ th node, which satisfies

$$
x_{n}=\sum_{m>n}^{N} f_{n, m} x_{m} .
$$

Corominas-Murtra et al. [13] showed that certain forms of $r_{n, m}$ give the power-law distribution of $x$. Here, we further expand upon sufficient conditions on $r_{n, m}$ to give rise to the power law.

We assume that $r_{n, m}$ is sampled from a certain independent random distribution and traces the cascade process on the realized network. Let $f(n, m)=\mathbb{E}\left[f_{n, m}\right]$ be the expectation value of $f_{n, m}$. We assume the network is large, i.e., $N \gg 1$. Based on this assumption, we approximate Eq. (3) by an integration,

$$
x(n) \approx \int_{n}^{N} f(n, m) x(m) d m,
$$

where $x(n)=\mathbb{E}\left[x_{n}\right]$.
Let us consider a class of $f(n, m)$ having the form

$$
f(n, m)=\frac{1}{m} g\left(\frac{n}{m}\right)
$$

with any non-negative function $g$ satisfying $\int_{0}^{1} g(t) d t=1$. This form represents the self-similarity of the edge weight distribution, and thus the network is considered as scalefree. Then, $x(n)=\eta n^{-1}$ is the solution of Eq. (4) with a normalization constant $\eta$. This result includes the particular case $r_{n, m} \propto n^{\alpha}$, which is reported to have the solution $x(n) \propto n^{-1}$ [14]. After the normalization, this case is reduced to $f(n, m)=(\alpha+1) / m(n / m)^{\alpha}$, which is one realization of Eq. (5). On the other hand, the reported counterexample $r_{n, m} \propto e^{-\beta n}$ [14] does not satisfy Eq. (5).

The constant $\eta$ can be evaluated by considering the total probability of the cascade proceeding from some node $m>n$ to a node $n^{\prime}<n$ (i.e., the total probability flux past $n \ll N)$ :

$$
1=\int_{0}^{n} d n^{\prime} \int_{n}^{N} \frac{1}{m} g\left(\frac{n^{\prime}}{m}\right) x(m) d m,
$$

which gives

$$
\frac{1}{\eta}=-\int_{0}^{1} \log t g(t) d t .
$$

Physically, $\eta$ can be understood as a measure of the preference for connecting to a closer node of the network. 
In the absence of any preference-for example, in the case $g(t)=1$-then we obtain $\eta=1$. If the connection between closer nodes is more probable $[g(t)$ is larger at $t \approx 1$ than at $t \approx 0$ ], then the integrand becomes smaller and $\eta>1$.

At times it may be convenient to consider the density distribution of $x$, as the explicit node ordering is not always clear. From $x(n)=\eta n^{-1}$, the number of nodes in the range $x \sim x+d x$ can be derived as

$$
\rho_{x}(x) d x=1 d n=\eta x^{-2} d x,
$$

which is another representation of Zipf's law with the index 1 .

Let us consider the joint distribution of $I$ and $t=n / m$, which is the number of edges within $I \sim I+d I$ and $t \sim t+d t$. This can be computed from the unit density of $n$ and $m$, and the technique of changing random variables,

$$
\rho_{I, t}(I, t) d I d t=1 d n d m=g(t) \frac{\eta}{2} I^{-2} d I d t .
$$

By integrating $\rho_{I, t}$ over $t$, we arrive at the power-law distribution of $I$,

$$
\rho_{I}(I)=\int_{0}^{1} \rho_{I, t}(I, t) d t=\frac{\eta}{2} I^{-2}
$$

In this derivation, we neglect the stochastic property of the edge weight and only consider the mean value of $f$. However, the random fluctuation of $f$ does not change the intensity distribution, as we show in the Supplemental Material [18].

Now, let us return to our particular system of interest, the $\gamma$-decay cascade of nuclei. Figure 1(a) shows a schematic illustration of the $\gamma$-decay cascade of ${ }^{77} \mathrm{Se}$ after a nuclear reaction, specifically, the thermal neutron capture of ${ }^{76} \mathrm{Se}$. In the nuclear case, $r_{n, m}$ is the physical transition rate from the $m$ th to the $n$th level, and thus $f_{n, m}$ is the branching ratio. $x_{m}$ is the total probability that the cascade passes through the $m$ th level, and hence $I_{n, m}=f_{n, m} x_{m}$ is the total probability of the transition $n \leftarrow m$ and is measured as the $\gamma$-ray intensity for each transition. The $\gamma$-ray spectrum has been measured for various kinds of reactions. In Fig. 1(b), we show the intensity and energy distribution of the $\gamma$ rays for the thermal neutron capture of ${ }^{76} \mathrm{Se}$, which are taken from the ENSDF database [16].

The distribution of the transition rates has been discussed for a long time, as this is a key parameter to explain the observed abundance of elements [19]. To consider this property of the $\gamma$-cascade spectrum, following an existing approach [20], let us start from the level density of a nucleus $\rho_{E}(E)$, i.e., the number of levels with a given excited energy $E$ per unit energy. In general, $\rho_{E}(E)$ is nearly exponentially dependent on $E$. A simple yet wellaccepted approximation thereof is the constant temperature model [21],

$$
\rho_{E}(E)=\rho_{0} e^{E / T}
$$

with $\rho_{0}=e^{-E_{0} / T} / T$, where $T$ parameterizes the leveldensity growth rate with excited energy (referred to as the "temperature" [21,22]), and $E_{0}$ is the energy backshift. Here, we assume that $T$ is constant over the entire energy range, and independent of quantum numbers, spin, and isospin. By integrating Eq. (11) from the ground state, the level index $n$ has the following relation to the level energy $E_{n}$ :

$$
E_{n}=T \log \left(\frac{n+\rho_{0} T}{\rho_{0} T}\right) .
$$

Note that the use of a different level-density model, such as the backshifted Fermi gas model, does not change the above relation, since all of them behave similarly in the energy range smaller than the resonance level.

The transition rates are expected to fluctuate according to the level pairs. On the other hand, the average rates are considered to depend on the transition energy but to be independent of the state [23-28]. In particular, the transition rate from state $m$ to state $n$ is often written as $2 \pi \omega_{n, m}^{3} \Gamma\left(\omega_{n, m}\right)$, where $\omega_{n, m}=E_{m}-E_{n}$ is the energy difference and $\Gamma\left(\omega_{n, m}\right)$ is the so-called gamma strength function. (Although we implicitly assume the dominance of electric or magnetic dipole transitions, the effect of quadrupole transitions can be absorbed by $\Gamma$.) As the energy dependence of the gamma strength function is not large, we may approximate the transition rate by taking its leading order:

$$
r(n, m) \propto\left(E_{m}-E_{n}\right)^{3}=\left[-T \log \left(\frac{n+\rho_{0} T}{m+\rho_{0} T}\right)\right]^{3} .
$$

This function has a $[-\log (n / m)]^{3}$ dependence with a large $m \gg \rho_{0} T$, whereas it is proportional to $(1-n / m)^{3}$ with small $m \ll \rho_{0} T$. Both of these satisfy Eq. (5), and so the $\gamma$ cascade spectrum is anticipated to very closely follow the power law. Using Eq. (7), we obtain $\eta=\frac{1}{4}$ for the case of large values of $m$, and $\eta=12 / 25$ for the case of small values of $m$.

Note that the detailed energy dependence of $\Gamma$, which has been discussed frequently, slightly changes the normalization value $\eta$ but not the power-law index. We have confirmed that reasonable profiles, such as the Lorentzian dependence of $\Gamma[24,29]$, the pygmy dipole resonances [30-32], and the low-energy enhancement $[33,34]$, do not change the value of $\eta$ significantly. Also note that the transition selection rules do not change the intensity distribution provided that the distributions of the total angular momentum number $J$ and the parity are uniform over the energy range, since this effect can be absorbed into fluctuations of $f$.

Figure 1(c) shows the intensity distribution of the $\gamma$ decay cascade for a thermal neutron capture of ${ }^{76} \mathrm{Se}$, which 


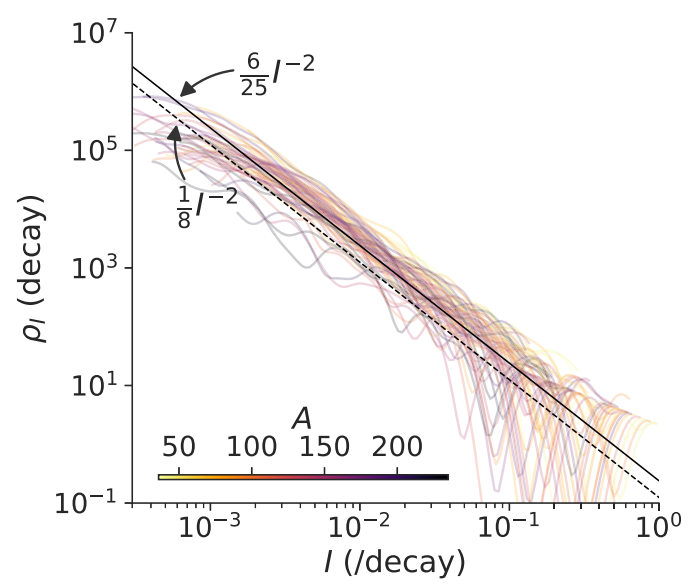

FIG. 2. Intensity histogram of $\gamma$-cascade spectra for all the reactions with more than $200 \gamma$-ray intensity entries in the ENSDF database [16]. The color of each line indicates the mass number of the nucleus $A$. The solid and dashed lines indicate Eq. (10) with $\eta=12 / 25$ and $1 / 4$, respectively.

is computed from the observed spectrum shown in Fig. 1(b) by the kernel density estimation method. The diagonal solid and dashed lines show the power law $(\eta / 2) I^{-2}$, with $\eta=$ $12 / 25$ and $\eta=\frac{1}{4}$, respectively. This experimental distribution is consistent with the theory.

In our subsequent investigations, we compared the results of the above model with those of a numerical simulation. RAINIER is a simulation tool for distributions of excited nuclear states and cascade fluctuations [35]. This tool adopts more exact nuclear properties, for example, the backshifted Fermi gas model is used for the level density, taking into account parity and angular momentum distributions, certain known low-lying energy levels, and the generalized Lorentzian form for the gamma strength function. The level-density parameters and gamma strength function parameters used in our simulation were taken from Refs. [22] and [36], respectively.

Figure 1(e) shows the simulated intensity-energy distribution of the $\gamma$-ray lines. This is consistent with the experiment [Fig. 1(b)], but the distribution is extended to the much weaker intensity side than the distribution observed experimentally. Figure 1(f) shows the simulated intensity distribution, which is very close to that predicted by Eq. (10) over more than 4 orders of magnitude. In the simulation, we can also compute the distribution of $x$ directly. Figure 1(d) shows $\rho_{x}(x)$, which we find is also consistent with the theory $\eta x^{-2}$, shown by diagonal lines.

To confirm the universality of this distribution, we generated intensity distributions for all $\gamma$-decay cascade spectra with more than $200 \gamma$-intensity entries in the ENSDF database [16]. Figure 2 shows the intensity distributions of these 69 experimentally observed $\gamma$-cascade spectra. The level-density parameters $T$ and $\rho_{0}$ and the symmetry of the nuclei analyzed in this work are also shown in Fig. 3(a), (b). As shown in Fig. 2, all the
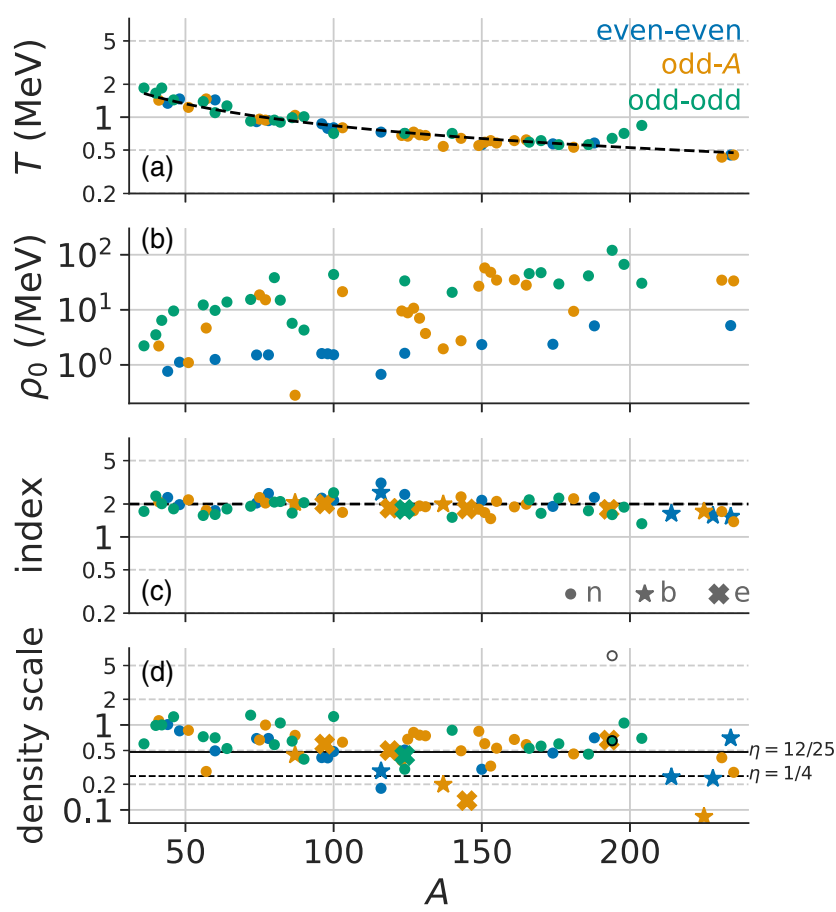

FIG. 3. Fitted results for the $\gamma$-ray intensity distribution. (a) Temperature parameters $T$ and (b) level-density scale $\rho_{0}$ for each nucleus [22]. (c) Index and (d) density scale of experimental $\gamma$-ray intensity distribution. The color of each marker indicates the type of nucleus and its shape (circle, star, or cross) indicates the reaction type (neutron capture, $\beta$ cascade, or electron capture, respectively). Although $T$ varies by a factor of 5 and $\rho_{0}$ varies by more than $10^{3}$, the index and density scale of the $\gamma$-ray intensity distribution are very close to the theoretical values ( 2 for the index and approximately $1 / 4-12 / 25$ for the density scale). The open circle in (d) shows the density scale for ${ }^{193}$ Ir from ENSDF, the intensities of which we found to be incorrect in the database by a factor of 10 . The result for the corrected spectrum is shown by a filled circle.

distributions are concentrated on a single straight line in the double-logarithmic graph. Equation (10) with $\eta=1 / 4$ and $\eta=12 / 25$ is plotted using black lines. The consistency is very clear over several orders of magnitude.

We estimated the distribution variation by fitting those shown in Fig. 2 with $\rho_{I}(I)=(a / 2) I^{-b}$, where $b$ and $a$ are adjustable parameters to be estimated from the distribution, index, and density scale, respectively. The results are shown in Fig. 3(c),(d). Although $T$ depends on the negative power of mass number $A\left(\approx A^{-2 / 3}[22]\right)$, which changes over a factor of 5 in this range, and $\rho_{0}$ varies over 3 orders of magnitude depending on the nuclear structure, the power-law index is concentrated in the small range $b=2.0 \pm 0.3$, which our theory predicts to be 2 . The density scale is distributed over the range $a=0.54 \pm 0.28$ per cascade, which is predicted to be approximately in the range $1 / 4 \sim 12 / 25$. The consistency with Eq. (10) indicates that the scale-free random network model robustly reflects the nature of the nuclear structure. Note that the 
finite-size effect of the network affects the intensity distribution to a certain extent but at the same time avoids the divergence of the total $\gamma$-ray photon number during the cascade (see the Supplemental Material for details).

Because Eq. (10) is a universal distribution and does not depend on the nuclear structure or reaction type, it can be used to roughly calibrate the $\gamma$-ray intensity. Indeed, our work revealed that the intensities of ${ }^{193}$ Ir stored in ENSDF are incorrect in that they differ from those reported in the original work [37] by a factor of 10 . This outlying behavior of this dataset is very obvious [Fig. 3(d)].

In this Letter, we have considered the pure decay process, in which the system can only transit to the lower levels without any excitation processes to the upper levels. By contrast, many-electron atoms in plasmas (for which the intensity power law was originally observed) can experience excitation. Corominas-Murtra et al. $[13,14]$ considered a similar process in which they mixed the decay process (consisting of only transits to lower levels) and the bidirectional jump process (in which excitation to higher levels could also occur in the system). They referred to this process as a noisy SSR process and showed that, by mixing these two processes, the exponent of the power law varies in the range 1-2. This is consistent with our explanation [5], where the exponent changes depending on the plasma parameters. This strongly suggests a direct connection between the atomic systems and the noisy SSR process. However, this relation would have to be further investigated in future.

In summary, we pointed out that the nuclear structure can be modeled using a scale-free random network. We showed that a random walk on a scale-free random acyclic network exhibits a power-law distribution for the number of edges with a given passing probability, which corresponds to the intensity of the $\gamma$ ray for the $\gamma$ cascade. All experimental $\gamma$-ray intensities stored in the ENSDF database adhere to this power law. Since this basic statistical property was derived using generic features of nuclei, a deviation from this law may be useful to study a specific feature of a particular nucleus.

This work was partly supported by JSPS KAKENHI Grant No. 19K14680, a grant from the Joint Research by the National Institutes of Natural Sciences (NINS) (NINS program no. 01111905), and partly by the Max-Planck Society for the Advancement of Science. J. C. B is supported by the Alexander von Humboldt Foundation. We thank José Crespo López-Urrutia, Wenjia Huang, and Hans Arwed Weidenmüller, for their useful discussions.

*Corresponding author. fujii@me.kyoto-u.ac.jp

[1] R. C. M. Learner, J. Phys. B 15, L891 (1982).

[2] C. Bauche-Arnoult and J. Bauche, J. Quant. Spectrosc. Radiat. Transfer 58, 441 (1997).
[3] J. Bauche, C. Bauche-Arnoult, and O. Peyrusse, Atomic Properties in Hot Plasmas (Springer International Publishing, Cham, 2015).

[4] J.-C. Pain, High Energy Density Phys. 9, 392 (2013).

[5] K. Fujii and J. C. Berengut, Phys. Rev. Lett. 124, 185002 (2020).

[6] We use $\epsilon_{0}$ in Ref. [5] instead of $T$.

[7] V. M. Yakovenko and J. B. Rosser, Rev. Mod. Phys. 81, 1703 (2009).

[8] M. V. Simkin and V. P. Roychowdhury, Phys. Rep. 502, 1 (2011).

[9] H. Kawamura, T. Hatano, N. Kato, S. Biswas, and B. K. Chakrabarti, Rev. Mod. Phys. 84, 839 (2012).

[10] D. Marković and C. Gros, Phys. Rep. 536, 41 (2014).

[11] M. A. Muñoz, Rev. Mod. Phys. 90, 031001 (2018).

[12] T. J. Perkins, E. Foxall, L. Glass, and R. Edwards, Nat. Commun. 5, 5121 (2014).

[13] B. Corominas-Murtra, R. Hanel, and S. Thurner, Proc. Natl. Acad. Sci. U.S.A. 112, 5348 (2015).

[14] B. Corominas-Murtra, R. Hanel, and S. Thurner, New J. Phys. 18, 093010 (2016).

[15] Pure and Applied Mathematics, edited by M. Lal Mehta (Elsevier Ltd, New York, 2004), Vol. 142, pp. 1-688.

[16] ENSDF database, http://www.nndc.bnl.gov/ensarchivals/, accessed:2019.

[17] B. Karrer and M. E. J. Newman, Phys. Rev. Lett. 102, 128701 (2009).

[18] See Supplemental Material at http://link.aps.org/ supplemental/10.1103/PhysRevLett.126.102502 for the effects of the stochasticity of the network structure as well as the resonance $\gamma$-decays.

[19] G. M. Tveten et al., The gamma-ray strength function of 89 Y and 90 Y, Technical Report, 2018, arXiv:1804.08109v1.

[20] K. Hagiwara et al., Prog. Theor. Exp. Phys. 2019, 023D01 (2019).

[21] T. Von Egidy, H. Schmidt, and A. Behkami, Nucl. Phys. A481, 189 (1988).

[22] T. von Egidy and D. Bucurescu, Phys. Rev. C 72, 044311 (2005).

[23] D. M. Brink, Some aspects of the interaction of light with matter, Ph.D. thesis, University of Oxford, 1955.

[24] P. Axel, Phys. Rev. 126, 671 (1962).

[25] M. Guttormsen, A. C. Larsen, A. Görgen, T. Renstrøm, S. Siem, T. G. Tornyi, and G. M. Tveten, Phys. Rev. Lett. 116, 012502 (2016).

[26] T. A. Brody, J. Flores, J. B. French, P. A. Mello, A. Pandey, and S. S. M. Wong, Rev. Mod. Phys. 53, 385 (1981).

[27] G. E. Mitchell, A. Richter, and H. A. Weidenmüller, Rev. Mod. Phys. 82, 2845 (2010).

[28] H. A. Weidenmüller and G. E. Mitchell, Rev. Mod. Phys. 81, 539 (2009).

[29] B. L. Berman and S. C. Fultz, Rev. Mod. Phys. 47, 713 (1975).

[30] A. Voinov, M. Guttormsen, E. Melby, J. Rekstad, A. Schiller, and S. Siem, Phys. Rev. C 63, 044313 (2001).

[31] M. Krtička, F. Bečvá, J. Honzátko, I. Tomandl, M. Heil, F. Käppeler, R. Reifarth, F. Voss, and K. Wisshak, Phys. Rev. Lett. 92, 172501 (2004).

[32] A. Simon, M. Guttormsen, A. C. Larsen, C. W. Beausang, P. Humby, J. T. Burke, R. J. Casperson, R. O. Hughes, 
T. J. Ross, J. M. Allmond, R. Chyzh, M. Dag, J. Koglin, E. McCleskey, M. McCleskey, S. Ota, and A. Saastamoinen, Phys. Rev. C 93, 034303 (2016).

[33] A. Voinov, E. Algin, U. Agvaanluvsan, T. Belgya, R. Chankova, M. Guttormsen, G. E. Mitchell, J. Rekstad, A. Schiller, and S. Siem, Phys. Rev. Lett. 93, 142504 (2004).
[34] S. Karampagia, B. A. Brown, and V. Zelevinsky, Phys. Rev. C 95, 024322 (2017).

[35] L. Kirsch and L. Bernstein, Nucl. Instrum. Methods Phys. Res., Sect. A 892, 30 (2018).

[36] H. I. Kim, M. J. Yi, and Y.-O. Lee, J. Nucl. Sci. Technol. 44, 1117 (2007).

[37] M. Balodis et al., Nucl. Phys. A641, 133 (1998). 